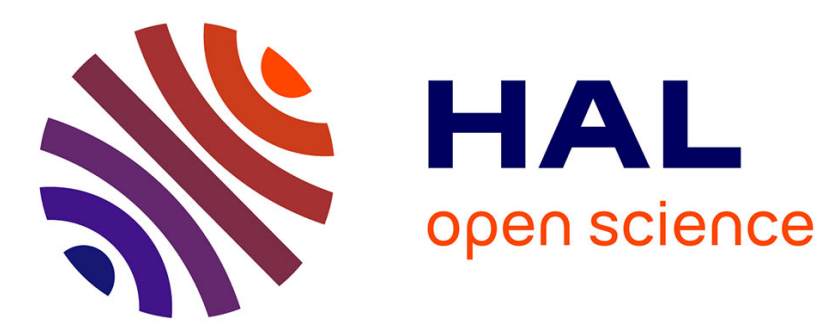

\title{
Speciations and Extinctions in a Self-Organizing Critical Model of Tree-Like Evolution
}

\author{
M. Kramer, N. Vandewalle, M. Ausloos
}

\section{To cite this version:}

M. Kramer, N. Vandewalle, M. Ausloos. Speciations and Extinctions in a Self-Organizing Critical Model of Tree-Like Evolution. Journal de Physique I, 1996, 6 (4), pp.599-606. 10.1051/jp1:1996232 . jpa-00247205

\section{HAL Id: jpa-00247205 https://hal.science/jpa-00247205}

Submitted on 1 Jan 1996

HAL is a multi-disciplinary open access archive for the deposit and dissemination of scientific research documents, whether they are published or not. The documents may come from teaching and research institutions in France or abroad, or from public or private research centers.
L'archive ouverte pluridisciplinaire HAL, est destinée au dépôt et à la diffusion de documents scientifiques de niveau recherche, publiés ou non, émanant des établissements d'enseignement et de recherche français ou étrangers, des laboratoires publics ou privés. 


\title{
Speciations and Extinctions in a Self-Organizing Critical Model of Tree-Like Evolution
}

\author{
M. Kramer $\left(^{*}\right)$, N. Vandewalle (**) and M. Ausloos $\left(^{* * *}\right)$ \\ S.U.P.R.A.S., Institut de Physique B5, Sart Tilman, Université de Liège, B-4000 Liège, Belgium
}

(Received 4 December 1994, revised 15 December 1995 and accepted 5 January 1996)

PACS.87.10.+e - General, theoretical and mathematical biophysics

PACS.05.40. $+\mathrm{j}-$ Fluctuation phenomena, random processes and Brownian motion

\begin{abstract}
We study analytically a simple model of a self-organızed critical evolution. The model considers both extinction and speciation events leading to the growth of phylogenetic-like trees. Through a mean-field like theory, we study the evolution of the local configurations for the tree leaves. The fitness threshold, below which life activity takes place through avalanches of all sizes is calculated. The transition between speciating (evolving) and dead trees is obtained and is in agreement with numerical simulations. Moreover, this theoretical work suggests that the structure of the tree is strongly dependent on the extinction strength.
\end{abstract}

Résumé. - Nous étudions analytiquement un modèle simple d'évolution auto-organısée critique. Le modèle considère des extinctions et des spécıations conduisant à une crosssance d'arbres phylogénétiques. Nous étudions ici par une théorıe de champ moyen l'évolution des configurations des extrémités de l'arbre. Le seuil critique de "fitness" en-dessous duquel des explosions d'activité biologique de toutes tailles se produisent est calculé. La transition entre arbres crosssants et arbres éteints est également obtenue en accord avec les simulations. En outre, ce travall théorique suggère que la structure des arbres générés dépend fortement du paramètre d'extinctions.

\section{Introduction}

Biological evolution is not considered as a continuous process but is thought to be like a succession of bursts of life activity separated by long perıods of quiescence on a geological timescale [1]. Such bursts seem to have all sizes including catastrophic ones like the Cambrian explosion some 570 million years ago.

In order to mimic the intermittency [2] of an evolving ecosystem, Bak and coworkers have untroduced an interesting model of evolution [3,4]. This model (BS) considers an ecosystem made of $N$ species arranged on a $d$-dimensionnal hypercubic lattice. A fitness $f_{2}$, which is a random number between zero and one, is assigned to the $i$ species. The higher the fitness 15, the more adapted is the species to its environment (the ecology). At each step of the

$\left({ }^{*}\right)$ e-mall address: kramer@gw.unipc.ulg.ac.be

$\left({ }^{* *}\right)$ e-mall address: vandewal@gw.unipc.ulg.ac.be

$\left({ }^{* * *}\right)$ Author for the correspondence (e-mall address: ausloos@gw.unipc.ulg.ac.be) 


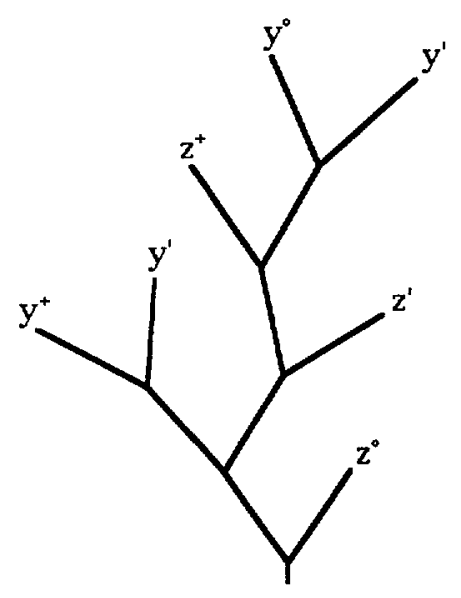

Fig. 1. - A small part of a phylogenetic-like tree. The species are labelled " $y$ " and " $z$ " with sublabels $\left({ }^{\prime}\right),\left({ }^{\circ}\right)$ and $\left({ }^{+}\right)$according to the different local configurations as discussed in the text.

evolution, the species having the minimum fitness value is selected and is supposed to make a so-called adaptative move by receiving a new random fitness value. Because of interactions in the ecosystem, this adaptative move is supposed to affect also the fitness values of neighbouring species. The evolution process generated by this model can be regarded as a particular random walk in a random medium. The interests of this model are 1) that from simple rules, it generates intermittent avalanches of all sizes, and ii) that the process is recognized to be self-organized critical [3], 1 e. to reach asymptotically an apparently critical steady-state (the so-called "evolution to the edge of chaos"). The latter dynamics is of physical interest [5] since various natural systems have a tendency to reach a critical self-organızed state like sandpiles [6], earthquakes [7], high- $T_{\mathrm{c}}$ superconductors [8],..

More recently, we have extended the BS model for an evolution allowing for a non-conservation of the number of species in the ecosystem [9-11]. The tree-like evolution model considers speciation and extinction events [11]. Each speciation corresponds to the differentiation of a species made of a large group of individuals in a population. The differentiation is considered to lead to a branching event. The formation of a tree-like structures is obtained with patterns in which the different species are located on the leaves (see Fig. 1). The "distance" $d_{m n}$ between two species ( $m$ and $n$ ) is the number of segments of the tree needed to connect one species $(m)$ to another $(n)$. A fitness $f_{\imath}$ which is a scalar random number between zero and one is associated to each $i$ living species. At each Monte-Carlo step, the less adapted species, i.e. the living $j$ species having the minımum fitness $f_{j}$ is selected. This species 1 s assumed to become extinct with an a priori probability $\exp \left(-f_{J} / r\right)$ or to give two differentiated species with a probability $\left\{1-\exp \left(-f_{\jmath} / r\right)\right\}$. The latter speciation event leads to two living species which both recelve a new arbitrary (uncorrelated here) fitness value. Of course, extinct species do not participate further to the evolution process. The parameter $r$ is hereby called the strength of the extinctions [11]. We also consider the presence of interactions between the species, such that the fitness $f_{2}$ of all the species which are separated by an arbitrary distance $d_{\imath \jmath}$ from the branching or dying species $j$ less than a short range $k$ are also updated with a new random number. This rule mimics the drastic change of the "fitness landscape" [12] within a range $k$ due to an evolution event. No correlation is introduced here between fitness values from ancestors to offsprings, or between offsprings. These effects could be examined in further work. 


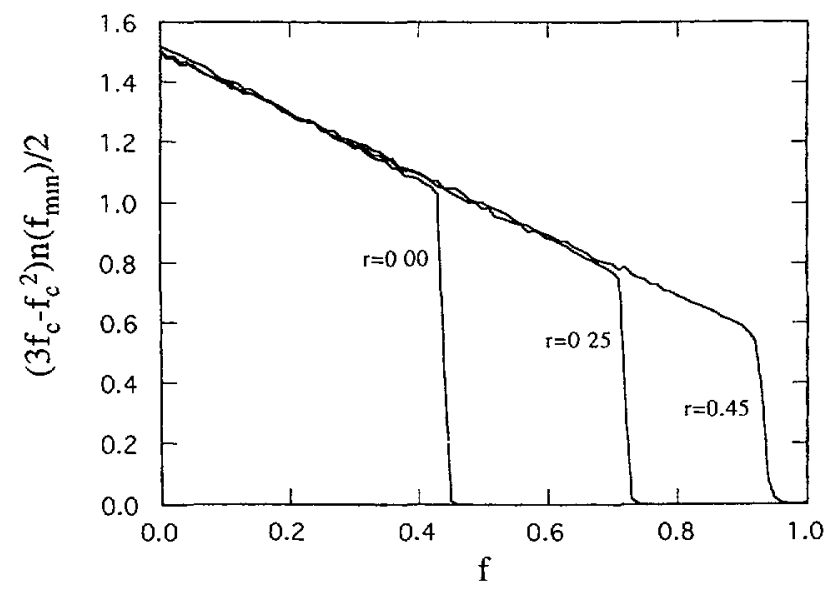

Fig. 2. - The distribution $n\left(f_{\min }\right)$ of the minimum fitness values $f_{\min }$ selected during the tree-like evolution process. Three different values of the extinction strength parameter $r$ are illustrated.

Moreover, we consider heren the case $k=2$ which is of biological interest since the majority of living species (the so-called "specialists" [13]) occupy narrow ecological niches and are only sensitive to their genetically nearest neighbourng species [13].

In the next section, we wll recall the most striking numerical results. In Section 3 , we theoretically investigate this evolution through an original study of the local configurations for the leaves of the tree. The critical threshold $f_{\mathrm{c}}(r)$ below which the life activity takes place is calculated and the transition between evolving and dead trees is obtained in agreement with the numerical results of Section 2. Finally, a conclusion will be drawn outlining the interest of these results and suggesting new developments.

\section{The Striking Numerical Results}

Let us recall the most important features presented by the above model as found from numerical work [11]. Without extinctions (for $r=0$ ), the model reduces to a previously studied model [9] for which the growth of trees never stops. As $r$ increases, the extinction events becomes more and more frequent such that the tree stops to grow above a certain value $r_{c}$. This critical value was numerically found to be $r_{\mathrm{c}}=0.48 \pm 0.01$ [11].

For $0 \leq r<r_{c}$, the evolution process reaches always an apparent steady-state. The latter is characterized by a step-like distribution of fitness values between a value $f_{c}$ and 1 . This characterizes the state of the majority of the species in the ecosystem. On the other hand, the fitness values $f_{\text {min }}$ of the selected species are distributed between 0 and $f_{c}$. The latter distribution $n\left(f_{\min }\right)$ is illustrated in Figure 2 for three different values of $r$. One should note that the distribution $n\left(f_{\mathrm{min}}\right)$ seems to be the sum of a rectangular and a triangular distribution. When $r>r_{\mathrm{c}}$, the process is limited in "time" since the tree growth stops.

Figure 3 presents $f_{c}$ as a function of the extinction strength $r$. For $r=0$, one finds the particular and non-trivial value $f_{\mathrm{c}}=0.445 \pm 0.010$ [9] which was theoretically predicted in a previous work [10]. For $r$ close to $r_{c}$, the threshold $f_{c}$ reaches 1 because the most adapted species only can resist against mutation. However, the $f_{\mathrm{c}}(r)$ curve falls to zero for $r$ close to 


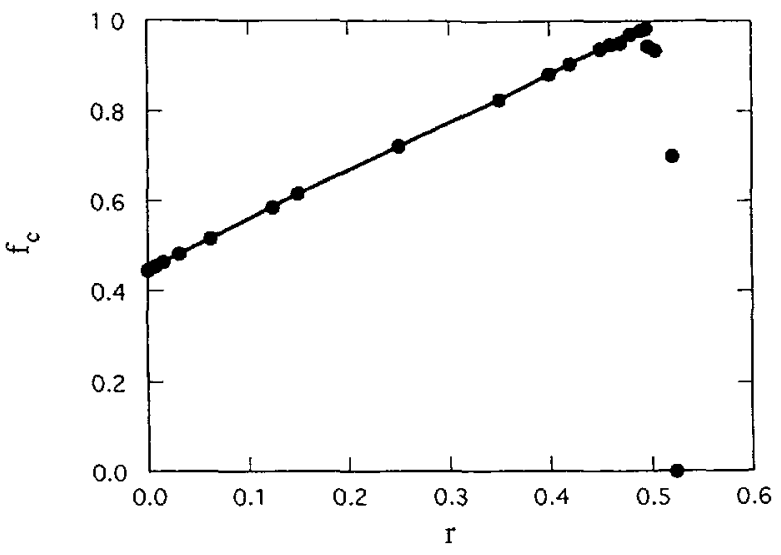

Fig. 3. - The threshold $f_{c}$ as a function of the extinction strength $r$. The continuous curve is the one obtained theoretically with the relations (2) and (6). Data points from reference [11].

$r_{\mathrm{c}}$ in Figure 3. This 1 s understood as resulting from the finite-size effect occurring close to $r_{\mathrm{c}}$. Above $r_{\mathrm{c}}$, the critical threshold $f_{\mathrm{c}}$ is rather meaningless since it is defined for living species only and for $r>r_{c}$ all species are after a finite "time" accumulated at $f=1$ and die.

The existence of such a threshold means that the whole life activity self-organizes for $0 \leq r<r_{c}$ and takes place below $f_{c}$. Physically, the attractor of this self-organization is thus a state where all species have a fitness above or equal to $f_{\mathrm{c}}$. This state is however unstable since the species with the minimum value (a species close to $f_{\mathrm{c}}$ ) will then be selected. Thus, some species having a fitness below $f_{\mathrm{c}}$ can appear later and can also induce some perturbation for the attractor of fitness distribution. Then, the new $f_{\imath}$ 's are chosen until the unstable state is again reached. This defines an avalanche as a connected sequence of biological activity (specrations and extinctions) below the threshold $f_{c}$, one observes an intermittency of avalanches of all sizes. The size-distribution of avalanches $n(s)$ is numerically found to be a power law $n(s) \sim s^{-\tau}$ with an exponent $\tau=3 / 2$ which seems to be independent of $r$ for $0 \leq r<r_{c}[11]$. This "no-scale" or power law distribution is the signature of self-organized critically [5] driving this evolution process.

Beside the self-organized critical dynamics of this process, it is of interest to observe the structure of the growing trees. The trees were numerically found to be scale invariant for $0 \leq r<r_{c}$ [11]. This is another slgnature of critically. However, the fractal dimension $D_{f}$ of the trees was thought to be dependent of the parameter $r$ [11]. This result was unexpected and is in contrast with classical phase transitions for which the value of the critical exponents does not change with any tuning of the relevant parameter. In fact, $D_{\mathrm{f}}$ was numerically found to decrease continuously from 2 (for $r=0$ ) towards 1 for $r \rightarrow r_{\mathrm{c}}$ [11]. This $r$-dependence is lllustrated in Figure 4 where three simulated trees are drawn for three different values of the extinction strength $r$. Each tree contains 2000 branching points. The variation of the tree structure from a two-dimensional growth for $r=0$ towards a filamentary-like growth for $r \approx r_{\mathrm{c}}$ is well marked.

In the following section, we theoretically investigate how the system evolves through local configurations and we extract theoretical values for $f_{\mathrm{c}}(r)$ and $r_{\mathrm{c}}$. 

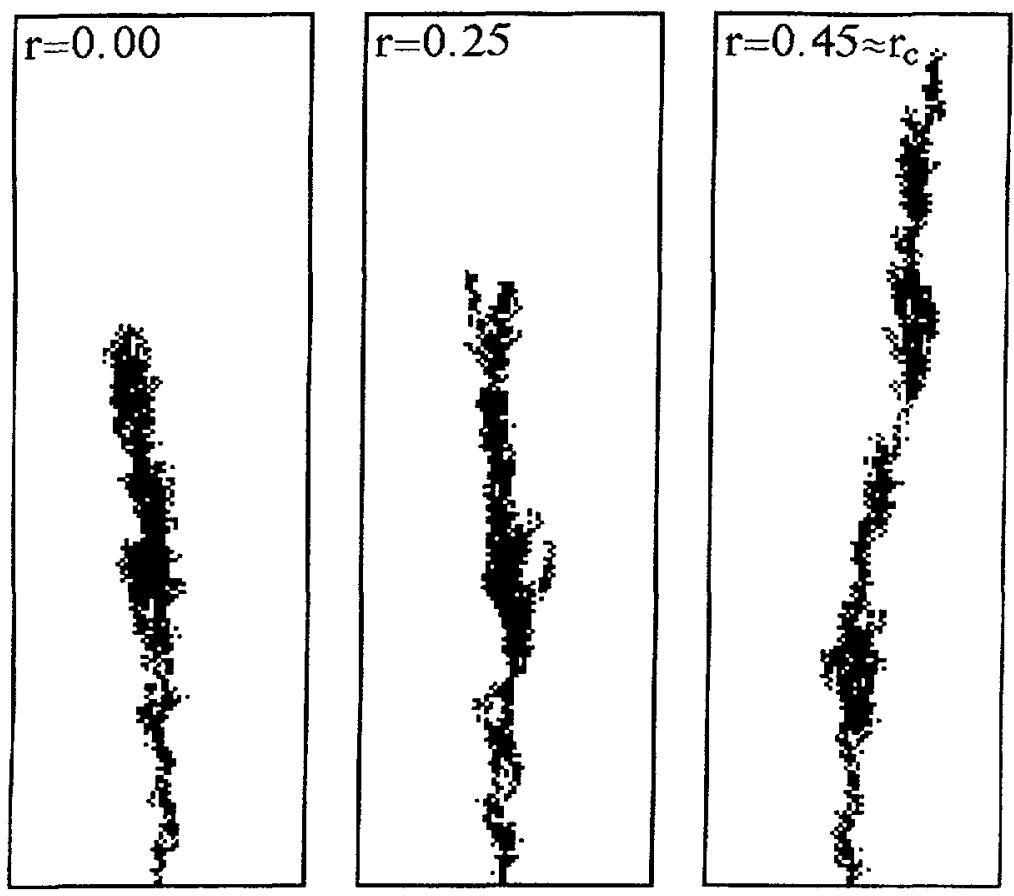

Fig. 4. - Three phylogenetic-like trees simulated for three different values of the extinction strength: $r=0.001, r=0.25$ and $r=0.45$ close to $r_{\mathrm{c}}$. Each tree is made of 2000 branching points.

\section{Theoretical Investigations}

For the $k=2$ process, two different types of local configurations can occur on the leaves. They are labelled " $y$ " and " $z$ " in Figure 1. The so-called $y$-species are coupled by pars, while the $z$-species are somewhat lonely. These different types of species lead to different speciation processes. Indeed, the speciation of a $y$-species gives a new parr of $y$-species branching out the selected species. This can also affect the nearest living neighbour species since the distance between the speciating species (the selected one) and the direct neighbouring $y$-species is equal to $k=2$. The latter becomes a $z$-species (see an example of $y$-speciation in Fig. 5). On the other hand, the speciation of a $z$-species cannot affect any other species since the distance between the speciating $z$-species and any other species is always greater than $k=2$.

The geometrical configurations of the tree-leaves, both $y$ - and $z$-species, can be put into three different classes: i) the class with living species having a fitness value less than $f_{c}$ such that these species could be selected by the process: they are active, 11) the class with living species having a fitness value larger than $f_{c}$ such that they cannot be selected for speciation or extinction: they are inactive, and iii) the dead species. For further developments, let us call $y^{\prime}\left(z^{\prime}\right)$ the active species, $y^{\circ}\left(z^{\circ}\right)$ the inactive species and $y^{+}\left(z^{+}\right)$the dead species.

To describe the evolution process only 9 different configurations of tree leaves as shown in Figure 6 have to be considered. They correspond to 6 parrs: $y^{\prime} y^{\prime}, y^{\prime} y^{\circ}, y^{\prime} y^{+}, y^{\circ} y^{\circ}, y^{\circ} y^{+}$and $y^{+} y^{+}$, as well as to $3 z$-configurations: $z^{\prime}, z^{\circ}$ and $z^{+}$One should note that 5 configurations do not participate in the evolution since they are dead or screened from the process. These blocked configurations are the $y^{+} y^{+}, y^{\circ} y^{\circ}$ and $y^{\circ} y^{+}$parrs as well as the $z^{\circ}$ and $z^{+}$species. 

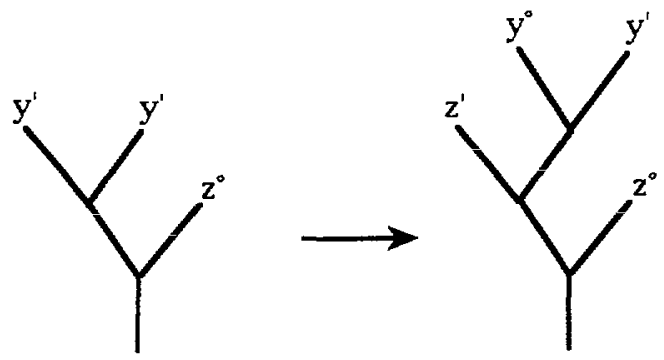

Fig. 5. - An example of evolution event occurring on a $y^{\prime}$-type leaf (on the top of the small branch). This example is a speciation leading to the formation of a new $y^{\circ} y^{\prime}$ pair and to a $z^{\prime}$-species. The probability of occurrence of the latter event is $(1-p)\left(1-f_{c}\right) f_{c}^{2}$. The state of the $z^{\circ}$-species on the bottom of the branch remains unchanged since the distance from the latter species to the specrating one is $3(>k)$.

(a)

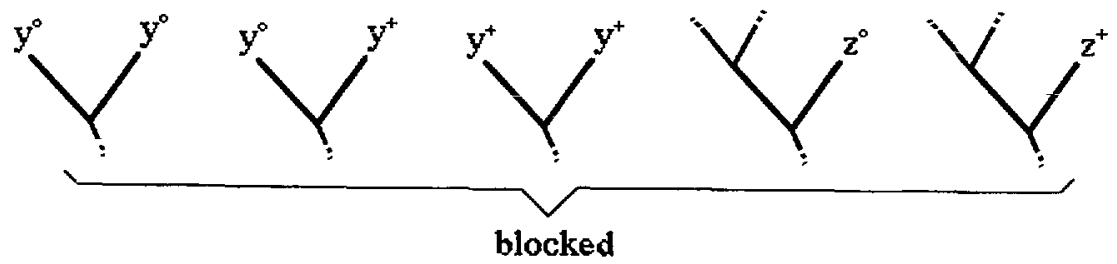

(b)

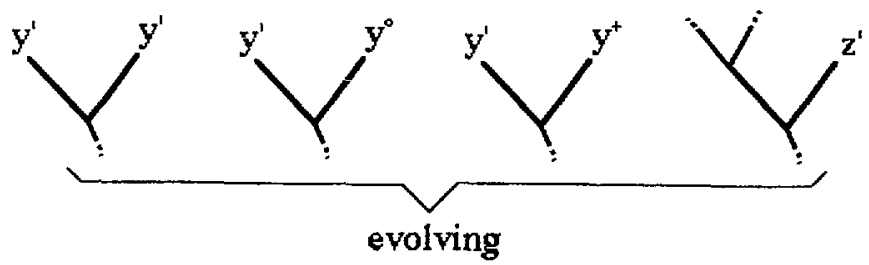

Fig. 6. - The 9 locally different configurations which are sufficient to describe the evolution process in our mean-field like theoretical development. One can distingush between the (a) blocked and the (b) evolving configurations with either one or two active species. By comparison to equations (1), one can easily see how the (b) cases can lead to new (b)-like configurations. The (b) configurations leading to (a) ones are irrelevant for describing the life process since such (a) configurations do not evolve anymore.

For a mean field-like approximation, we can assume that all the active species speciate or become extinct in a single mean-field time step $t$. One should note that the latter time is not equivalent to the Monte-Carlo time. In order to keep some essence of the process, we also consider the restriction that for the particular $y^{\prime} y^{\prime}$ configuration only one species speciates or becomes extinct. A possible evolution of a $y^{\prime} y^{\prime}$ configuration is lllustrated in Figure 5 as an example. The latter particular example is a speciation leading to a new $y^{\circ} y^{\prime}$ pair and to a $z^{\prime}$ species. Let $p$ be the probability that a chosen species becomes extinct $(+)$. One should note that the probability to have a new living and active species $\left({ }^{\prime}\right)$ is $(1-p) f_{\mathrm{c}}$ while the probability to have a new living and inactive species $\left(^{\circ}\right)$ is $(1-p)\left(1-f_{c}\right)$. One can write 
the mean-field evolution for the number of configurations participating to the process through recurrence relations:

$$
\begin{aligned}
N_{y^{\prime} y^{\prime}}(t+1) & =(1-p) f_{\mathrm{c}}^{2}\left[N_{y^{\prime} y^{\prime}}(t)+N_{y^{\prime} y^{\circ}}(t)+N_{y^{\prime} y^{+}}(t)+N_{z^{\prime}}(t)\right] \\
N_{y^{\circ} y^{\prime}}(t+1) & =2(1-p) f_{\mathrm{c}}\left(1-f_{\mathrm{c}}\right)\left[N_{y^{\prime} y^{\prime}}(t)+N_{y^{\prime} y^{\circ}}(t)+N_{y^{\prime} y^{+}}(t)+N_{z^{\prime}}(t)\right] \\
N_{y^{+} y^{\prime}}(t+1) & =p f_{\mathrm{c}}\left[N_{y^{\prime} y^{\prime}}(t)+N_{y^{\prime} y^{\circ}}(t)\right] \\
N_{z^{\prime}}(t+1) & =(1-p) f_{\mathrm{c}}\left[N_{y^{\prime} y^{\prime}}(t)+N_{y^{\prime} y^{\circ}}(t)\right]
\end{aligned}
$$

where the factors are probabilities for creating such configurations. One can find that the above system satisfies a steady-state solution only for

$$
(1-p)\left(f_{\mathrm{c}}^{3}-f_{\mathrm{c}}^{2}-2 f_{\mathrm{c}}\right)+1=0
$$

The probability $p$ is a function of both $r$ and $f_{\mathrm{c}}$. One should remark that the case $p=0$ is equivalent to consider only speciations $(r=0)$ without extinctions. Then, the relation (2) reduces to the relation found in reference [10] having a single root between 0 and 1, i.e. a threshold at $f_{\mathrm{c}} \approx 0.445$.

In order to find a relation between $f_{\mathrm{c}}$ and the extinction strength $r$, one has to calculate the probability $p$ for extincting a selected species. In doing so, one needs to know the distribution for the whole set of minımum fitness values $n\left(f_{\min }\right)$, i.e. the distribution of the selected species. Indeed, $p$ is given by

$$
p=\int_{0}^{1^{\prime}} n\left(f_{\min }\right) \exp \left(-\frac{f_{\min }}{r}\right) d f_{\min }
$$

For the BS model, the $n\left(f_{\min }\right)$ distribution was found to be linear vamshing to zero at $f \approx f_{\mathrm{c}}$ $[3,4]$. However, the present model shows a different $n\left(f_{\min }\right)$ distribution which seems to be the sum of a triangular and a rectangular distribution as noted in Section 2. This difference is understood from the fact that each speciation event in the present model creates new offsprings with new uniformly distributed random fitness values. This corresponds to add a rectangular (flat) distribution on the triangular distribution of the BS model.

For $r \approx r_{c}$, the distribution for the minimum fitness values is found to be linear

$$
n\left(f_{\min }\right)=3 / 2-f_{\min }
$$

with a slope -1 . Moreover, for all $0 \leq r<r_{c}$ values, the $n\left(f_{\min }\right)$ distribution collapses for $f_{\min }<f_{\mathrm{c}}(r)$ on the above distribution $3 / 2-f_{\min }$ using an approprate scaling factor. This 1s shown in Figure 2. The normalization for $0 \leq f_{\min } \leq f_{\mathrm{c}}(r)$ of the $n\left(f_{\min }\right)$ distribution of equation (4) gives

$$
\left\{\begin{array}{l}
n\left(f_{\min }\right)=\frac{3-2 f_{\min }}{3 f_{\mathrm{c}}-f_{\mathrm{c}}^{2}}, \text { for } f_{\min } \text { below } f_{\mathrm{c}} \\
n\left(f_{\min }\right)=0, \text { for } f_{\min } \text { above } f_{\mathrm{c}}
\end{array}\right.
$$

Using the latter $a d$ hoc distribution $n\left(f_{\min }\right)$, one can find the extinction probability

$$
p=\frac{r}{3 f_{\mathrm{c}}-f_{\mathrm{c}}^{2}}\left[(3-2 r)\left(1-\exp \left(-\frac{f_{c}}{r}\right)\right)+2 f_{\mathrm{c}} \exp \left(-\frac{f_{\mathrm{c}}}{r}\right)\right]
$$

Using this definition for $p$ in equation (2) and searching numerically for the solution of the resulting transcendental equation, one finds $f_{c}(r)$. The result is drawn in Figure 3 . This curve is roughly linear in the range of interest. Moreover, the agreement with the numerical values is quite good as seen in Figure 3. Since $f_{c}\left(r_{c}\right)=1$, one can then deduce the critical extinction strength $r_{\mathrm{c}}$ for growing or dead trees. Our theoretical estimate gives $r_{\mathrm{c}} \approx 0.495$ which is also in quite good agreement with the numerical value $r_{\mathrm{c}}=0.48 \pm 0.01[10]$. 


\section{Conclusion}

In the preceding section, we have described the evolution process for leaf configurations. Two hypotheses have been made: 1) correlations are neglected even though more than one active species is selected at each step for describing the process through the set of equations (1), and ii) the distribution $n\left(f_{\min }\right)$ is ad hoc. The present mean-field approximation can thus be considered to be variationally based.

However, the agreement is quite remarkable. This method is thus useful for describing such a kind of self-organized critical processes.

A further comment is however in order. As pointed out in Section 2, the most striking result for the tree evolution model is the unexpected variation of the fractal dimension $D_{\mathrm{f}}$ of the trees with the parameter $r$. The present theoretical investigations cannot predict the global structure of the growing tree since the mean-field time $t$ cannot be related to any geometrical property of the tree. However, our theoretical investigations show also that the local structure of the tree (leaf configurations) is strongly dependent on the extinction strength $r$ (see the set of Eqs. (1)). Further work should be made in order to test whether $D_{\mathrm{f}}$ vary with $r$.

\section{Acknowledgments}

Part of this work was financially supported through the Impulse Program on High Temperature Superconductors of the Belgium Federal Services for Scientific, Technical and Cultural (SSTC) Affairs under contract SU/02/013. This work was also supported through the ARC (94-99/174) contract of the University of Liège. N.V. thanks the Belguum Research Funds for Industry and Agriculture (FRIA, Brussels). A special grant from FNRS/LOTTO allowed us to perform specific numerical work.

\section{References}

[1] Gould J., Paleobiology 3 (1977) 115; Eldregde N. and Gould S.J., Nature 332 (1988) 211.

[2] Bergé P., Pomeau Y. and Vidal Ch., L'ordre dans le chaos (Hermann, Paris, 1984).

[3] Bak P. and Sneppen K., Phys. Rev. Lett. 71 (1993) 4083.

[4] Flyvbjerg H., Sneppen K. and Bak P., Phys. Rev. Lett. 71(1993) 4087.

[5] Bak P., Chen K. and Creutz M., Nature 342 (1989) 780.

[6] Dhar D. and Ramaswamy R., Phys. Rev. Let.t. 63 (1989) 1659.

[7] Sornette A. and Sornette D., Europhys. Lett. 9 (1989) 197.

[8] Pla O. and Nor1 F., Phys. Rev. Lett. 67 (1991) 919.

[9] Vandewalle N. and Ausloos M., J. Phys. I 5 (1995) 1011.

[10] Vandewalle N. and Ausloos M., Physıca D 90 (1996) 262.

[11] Vandewalle N. and Ausloos M., Europhys. Lett. 32 (1995) 613.

[12] Zhang Y.C., Phys. Rev. Lett. 56 (1986) 2113.

[13] Lewin R., Science 210 (1980) 883. 